\title{
Using body mass index to estimate individualised patient radiation dose in abdominal computed tomography
}

\author{
Siobhan O'Neill ${ }^{1,2}$, Richard G. Kavanagh ${ }^{1,2^{*}}$ (D), Brian W. Carey ${ }^{2}$, Niamh Moore ${ }^{1}$, Michael Maher ${ }^{1,2,3}$ \\ and Owen J. O'Connor ${ }^{1,2,3}$
}

\begin{abstract}
Background: The size-specific dose estimate (SSDE) is a dose-related metrics that incorporates patient size into its calculation. It is usually derived from the volume computed tomography dose index $\left(\mathrm{CTDI}_{\text {vol }}\right)$ by applying a conversion factor determined from manually measured anteroposterior and lateral skin-to-skin patient diameters at the midslice level on computed tomography (CT) localiser images, an awkward, time-consuming, and not highly reproducible technique. The objective of this study was to evaluate the potential for the use of body mass index (BMI) as a size-related metrics alternative to the midslice effective diameter $\left(D_{E}\right)$ to obtain a size-specific dose (SSDE) in abdominal $C T$.

Methods: In this retrospective study of patients who underwent abdominal CT for the investigation of inflammatory bowel disease, the $D_{E}$ was measured on the midslice level on CT-localiser images of each patient. This was correlated with patient $\mathrm{BMI}$ and the linear regression equation relating the quantities was calculated. The ratio between the internal and the external abdominal diameters $\left(D_{\text {RATIO }}\right)$ was also measured to assess correlation with radiation dose. Pearson correlation analysis and linear regression models were used.
\end{abstract}

Results: There was good correlation between $D_{E}$ and patient BMI $(r=0.88)$. An equation allowing calculation of $D_{E}$ from $B M I$ was calculated by linear regression analysis as follows: $D_{E}=0.76(B M I)+9.4$. A weak correlation between radiation dose and $D_{\text {RATIO }}$ was demonstrated $(r=0.45)$.

Conclusions: Patient BMI can be used to accurately estimate $D_{E}$ obviating the need to measure anteroposterior and lateral diameters in order to calculate a SSDE for abdominal CT.

Keywords: Abdomen, Body mass index, Tomography (x-ray, computed), Radiation dosage

\section{Key points}

- A strong correlation between BMI and $D_{E}$ was found

- BMI can be used to estimate patient $D_{E}$

- Calculating patient BMI can facilitate individualised patient radiation dose estimation in abdominal CT

\section{Background}

The individual patient radiation dose from computed tomography $(\mathrm{CT})$ is notoriously difficult to estimate. There is a growing interest in this topic, however, due to

\footnotetext{
* Correspondence: richykav@gmail.com

${ }^{1}$ Department of Radiology, Cork University Hospital, Wilton, Cork, Ireland

2Department of Radiology, University College Cork, Cork, Ireland

Full list of author information is available at the end of the article
}

the ever-increasing CT use and general concerns about the risks associated with radiation exposure from medical imaging. This has precipitated the increased use of dose monitoring systems in clinical practice.

Current CT scanner radiation dose output following patient imaging is displayed in the CT dose report in terms of volume CT dose index $\left(\mathrm{CTDI}_{\mathrm{vol}}\right)$ and dose length product (DLP), standardised measurements deduced from homogenous phantoms under normalised conditions $[1,2]$. These parameters do not provide a direct measure of the individualised patient radiation dose, a variable that is dependent on patient size.

The size-specific dose estimate (SSDE) is a dose-related metrics that incorporates patient size into its calculation. This metric has been advocated for the reporting of 
patient radiation dose in $\mathrm{CT}$ by the American Association of Physicists in Medicine (AAPM) task group 204 and has increasingly been applied and accepted $[3,4]$. The SSDE is derived from the CTDI $\mathrm{I}_{\mathrm{vol}}$ by applying a conversion factor determined from manually measured anteroposterior and lateral skin-to-skin patient diameters at the midslice level on CT-localiser images [3]. On a practical level this technique can be awkward and time-consuming, and open to interobserver measurement variability.

The objective of this study was to evaluate the potential for using body mass index (BMI) as an alternative size metrics, in lieu of measured body diameters, to estimate patient effective diameter $\left(D_{E}\right)$. Thus, estimation of SSDE at the time of CT scanning would be more user-friendly, contributing positively to patient radiation dose optimisation.

\section{Methods}

\section{Subjects}

This study was performed retrospectively on CT data acquired as part of a clinical trial protocol investigating the use of CT in inflammatory bowel disease (ClinicalTrials. gov Identifier: NCT 01244386) [5] with approval from the institution Clinical Research Ethics Committee. Fifty adult patients were included in this study and, as part of this trial protocol, all patients signed informed consent.

\section{CT scan protocol}

All patients underwent a CT scan of the abdomen and pelvis with a standardised protocol using the following parameters: scan range encompassing the lung bases to the pubic symphysis; 0.625 -mm slice acquisition thickness; intravenously administered contrast (Iohexol, Omnipaque 300, General Electric Healthcare, Waukesha, WI, USA) delivered at $2.5 \mathrm{~mL} / \mathrm{s}$ and imaged in the portal venous phase; $1.5 \mathrm{~L}$ of positive oral contrast (2\% Gastrografin, Bracco Diagnostics Inc., Princeton, NJ, USA); tube voltage of $120 \mathrm{kVp}$; automated tube current modulation resulting in a variable current with a minimum of $50 \mathrm{~mA}$ and a maximum of $350 \mathrm{~mA}$; gantry rotation time of $0.8 \mathrm{~s}$; noise index 38. All CT images were acquired using a single 64-slice multi-detector row CT scanner (Lightspeed VCT-XTe, GE Healthcare, General Electric Medical Systems, Waukesha, WI, USA). The DLP and CTDI ${ }_{\text {vol }}$ values, as well as the corresponding phantom size, were recorded from each CT dose report. CTDI ${ }_{\mathrm{vol}}$ and DLP tolerances were verified using a standard $32-\mathrm{cm}$ Perspex phantom, a $10-\mathrm{cm}$ ionisation chamber with a Victoreen NERO $\mathrm{mAx}$ unit (Fluke Biomedical, Solon, OH, USA).

The SSDEs were calculated by multiplying the CTDI ${ }_{\mathrm{vol}}$ of each patient by conversion factors corresponding to the effective patient diameters in the AAPM reference tables [3]. The imaging performance and assessment from CT patient dosimetry calculator (ImPACT version 0.99x, London, UK) was used to calculate the effective dose.

\section{BMI measurement}

Each patient had weight and height measurements performed and their BMI calculated immediately prior to CT scan using a dedicated calibrated measuring device (electronic measuring station Model 763, Seca Medical, Hamburg, Germany). BMI data were used to subdivide patient groups, where underweight referred to $\mathrm{BMI}<$ $18.5 \mathrm{~kg} / \mathrm{m}^{2}$, normal weight referred to $18.5 \leq \mathrm{BMI}<$ $25 \mathrm{~kg} / \mathrm{m}^{2}$, overweight referred to $25 \leq \mathrm{BMI}<30 \mathrm{~kg} / \mathrm{m}^{2}$ and obese referred to $\mathrm{BMI} \geq 30 \mathrm{~kg} / \mathrm{m}^{2}$.

\section{Body diameter measurements}

Images were reviewed on a picture-archiving and communication system (PACS) workstation (Impax 6.3.1,

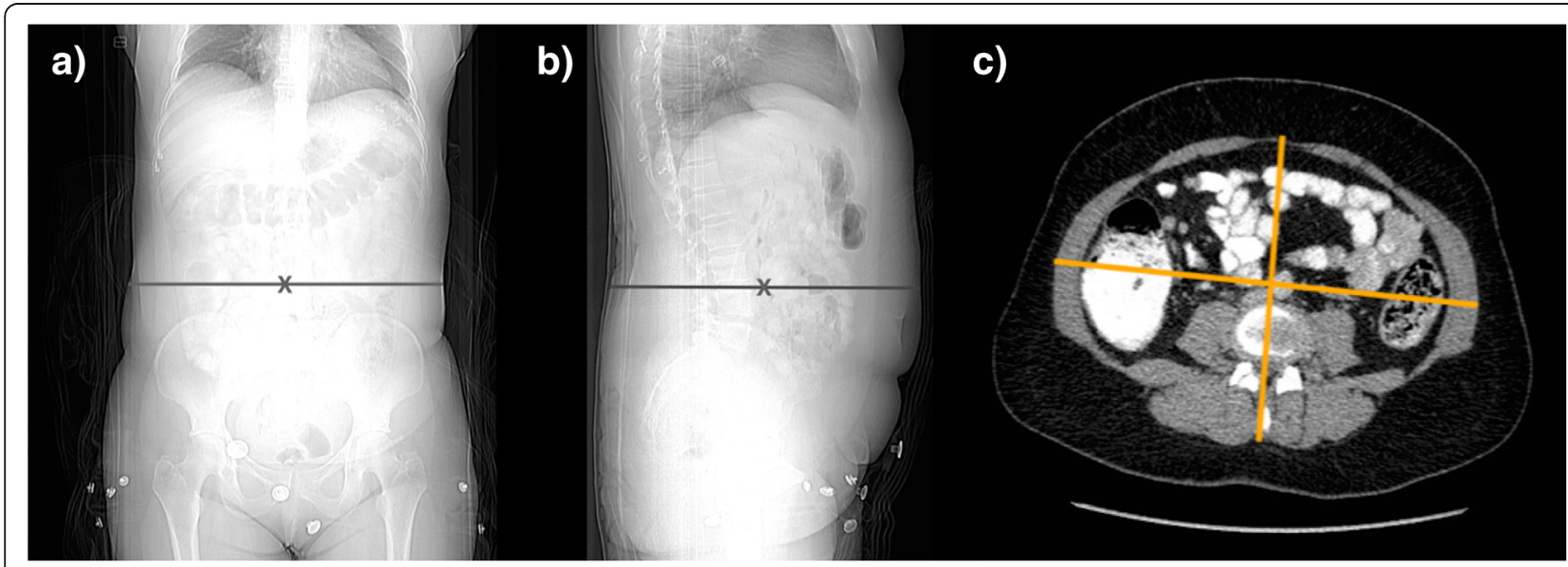

Fig. 1 Measurement of lateral $\left(D_{L A T}\right)(\mathbf{a})$ anteroposterior $\left(D_{A P}\right)(\mathbf{b})$ skin-to-skin patient diameters at the midslice level on $C T$-localiser images. Measurement of the inner lateral and anteroposterior diameters on the axial midslice CT image excluding the subcutaneous adipose tissue (c) to allow calculation of the effect inner diameter $\left(D_{I N}\right)$ and the effective diameter ratio $\left(D_{R A T I O}\right)$ 
Table 1 Summary of mean values for computed tomography dose metrics, overall and stratified for body mass index (BMI)

\begin{tabular}{lllll}
\hline & All $(n=50)$ & BMl $<25 \mathrm{~kg} / \mathrm{m}^{2}(n=32)$ & BMl $\geq 25 \mathrm{~kg} / \mathrm{m}^{2}(n=18)$ & $p$-value \\
\hline CTDI $_{\text {vol }}$ (mGy) & $6.26 \pm 3.83$ & $4.33 \pm 0.83$ & $9.68 \pm 4.65$ & $0.000^{\mathrm{a}}$ \\
DLP (mGy.cm) & $299.42 \pm 196.06$ & $202.36 \pm 41.27$ & $471.96 \pm 241.73$ & $0.000^{\mathrm{a}}$ \\
SSDE (mGy) & $7.81 \pm 3.08$ & $6.22 \pm 0.75$ & $10.64 \pm 3.61$ & $0.000^{\mathrm{a}}$ \\
Effective dose (mSv) & $4.77 \pm 3.23$ & $3.18 \pm 0.62$ & $7.59 \pm 1.64$ & $0.000^{\mathrm{a}}$ \\
\hline
\end{tabular}

$C T D I_{\text {vol }}$ volume-computed tomography dose index, DLP dose length product, SSDE size-specific dose estimate, BMI Body mass index

Data as means \pm standard deviations of the mean

aalue shows a statistically significant difference with a two-tailed $p$-value of less than 0.05 , when the radiation doses of each protocol are compared with one another

AGFA Healthcare, Morstel, Belgium) in a DICOM format. As per AAPM Report 204 guidelines, body diameters were measured at the midslice level (median image of the craniocaudal scanning length) on the CT-localiser images because, for larger patients, maximum skin-to-skin distance is often not included on transverse CT images $[3,6]$. Diameter measurements were performed manually with the electronic callipers available on the PACS using a window width of 350 Hounsfield Units (HU) and window level of $50 \mathrm{HU}$. From personal experience at our institution, analysis of interoperator variability for PACS-based anthropometric measurements shows no statistically significant differences. Therefore, a single investigator carried out all measurements. A fixed window level and setting was used for each individual study.

Maximum skin-to-skin anteroposterior diameter $\left(D_{A P}\right)$ and lateral diameter $\left(D_{L A T}\right)$ were measured in centimetres on lateral and anteroposterior localiser images, respectively. $D_{A P}$ is defined as the anteroposterior skin-to-skin diameter on the lateral localiser at the midslice level (Fig. 1a) while $D_{L A T}$ is defined as the lateral skin-to-skin diameter on the anteroposterior localiser image at the midslice level (Fig. 1b). The $D_{E}$ is defined as the diameter of the circle with area equivalent to the cross-sectional area of the patient at the particular z-axis level (i.e. the midslice level) and

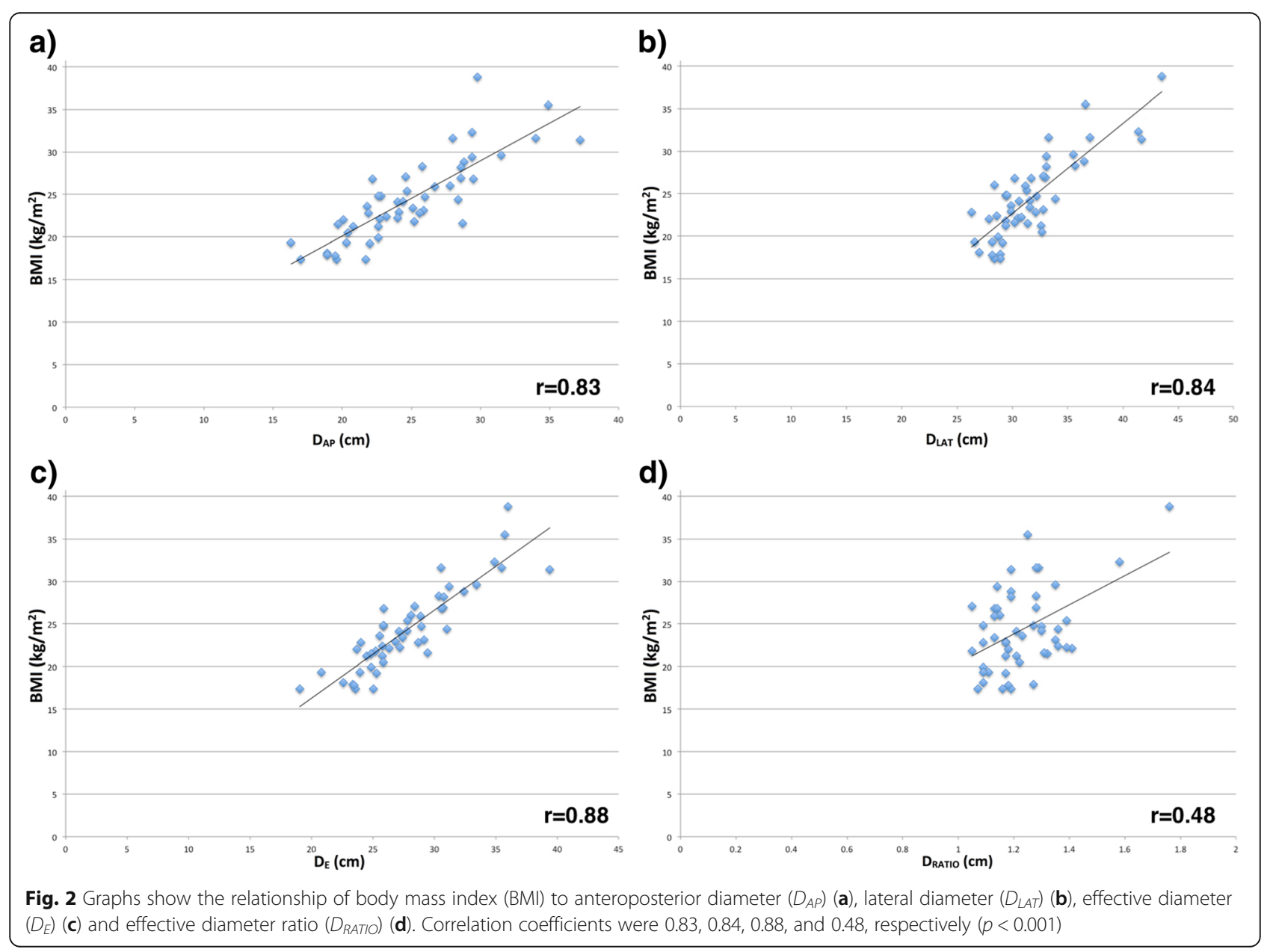


Table 2 Summary of body mass index category and midslice diameter measurements

\begin{tabular}{lllll}
\hline & $D_{A P}(\mathrm{~cm})$ & $D_{\text {LAT }}(\mathrm{cm})$ & $D_{E}(\mathrm{~cm})$ & $D_{\text {RATIO }}$ \\
\hline Overall $(n=50)$ & $24.77 \pm 4.53$ & $31.53 \pm 3.9$ & $27.79 \pm 4.12$ & $1.23 \pm 0.13$ \\
Underweight $(n=6)$ & $19.27 \pm 1.52$ & $27.42 \pm 2.23$ & $22.85 \pm 2.03$ & $1.16 \pm 0.07$ \\
Normal weight $(n=26)$ & $23.12 \pm 2.74$ & $30.23 \pm 1.92$ & $26.22 \pm 2.16$ & $1.22 \pm 0.11$ \\
Overweight $(n=12)$ & $27.35 \pm 2.62$ & $32.71 \pm 2.37$ & $29.88 \pm 2.13$ & $1.2 \pm 0.1$ \\
Obese $(n=6)$ & $32.22 \pm 3.65$ & $38.92 \pm 3.89$ & $35.34 \pm 2.84$ & $1.39 \pm 40.23$ \\
\hline
\end{tabular}

$B M I$ body mass index; data as means \pm standard deviations of the mean

Underweight referred to $B M I<18.5 \mathrm{~kg} / \mathrm{m}^{2}$, normal weight referred to $18.5 \leq \mathrm{BMI}<25 \mathrm{~kg} / \mathrm{m}^{2}$, overweight referred to $25 \leq \mathrm{BMI}<30 \mathrm{~kg} / \mathrm{m}^{2}$, obese referred to $\mathrm{BMI} \geq 30 \mathrm{~kg} / \mathrm{m}^{2}$
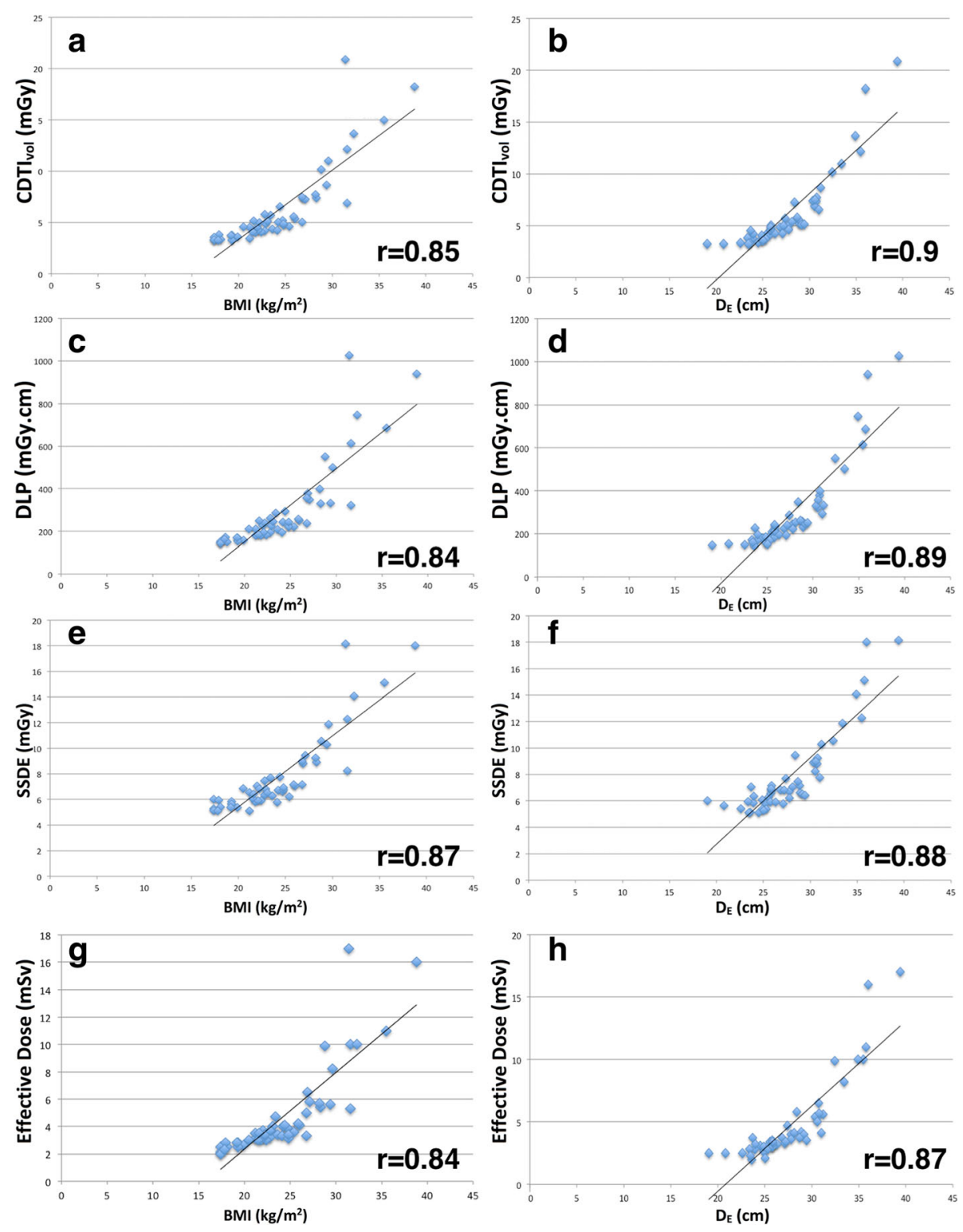

Fig. 3 Scatterplots show: volume-computed tomography dose index (CTDI $\left.{ }_{\text {vol }}\right)$ for body mass index (BMI) (a) and effective diameter (b) with correlation $r$ values of 0.85 and 0.9 , respectively; dose length product (DLP) for BMI (c) and effective diameter (d) with $r$ correlation values of 0.84 and 0.89 , respectively; size-specific dose estimate (SSDE) for BMI (e) and effective diameter ( $\mathbf{f}$ ) with $r$ correlation values of 0.87 and 0.88 , respectively; effective dose for BMI ( $\mathbf{g}$ ) and effective diameter (h) with $r$ correlation values of 0.84 and 0.87 , respectively 
is calculated as the geometric mean of $D_{A P}$ and $D_{L A T}$, as follows:

$$
D_{E}=\sqrt{ }\left(D_{A P} \times D_{L A T}\right)
$$

The outer $D_{E}\left(D_{\text {OUT }}\right)$ equates to the conventional $D_{E}$ calculated using the AAPM method described above. The inner $D_{E}\left(D_{I N}\right)$ is derived using the anteroposterior $\left(D_{A P(I N)}\right)$ and lateral diameters $\left(D_{L A T(I N)}\right)$ measured on an axial CT image at the midslice level, excluding the subcutaneous adipose tissue (Fig. 1c). The $D_{I N}$ is then calculated as the geometric mean of $D_{A P(I N)}$ and $D_{L A T(I N)}$, as follows:

$$
D_{I N}=\sqrt{ }\left(D_{A P(I N)} \times D_{L A T(I N)}\right)
$$

The $D_{E}$ ratio $\left(D_{R A T I O}\right)$, another patient size-related metrics proposed by Lamoureux et al. [7], was also calculated, as follows:

$$
D_{\text {RATIO }}=D_{\text {OUT }} / D_{I N}
$$

\section{Statistical analysis}

Data were collated using Microsoft Excel 2010 (Microsoft Corporation, Redmond, WA, USA) and statistical analyses were conducted by using Microsoft Excel 2010 and GraphPad Prism version 5.0 (GraphPad Software Inc., San Diego, CA, USA). Descriptive statistics including means, standard deviations and ranges were calculated. BMI, dose indices $\left(\mathrm{CTDI}_{\mathrm{vol}}\right.$, DLP, SSDE, effective dose) and body diameters $\left(D_{A B} D_{L A T}, D_{A P+} D_{L A T}, D_{E}, D_{R A T I O}\right)$ were recorded for each patient CT examination. The correlations between BMI, dose indices, and body diameter measurements were examined with Pearson correlation analysis $(r)$. Linear regression models were used to assess the dependence of CTDI, DLP, SSDE, and effective dose on BMI. Linear regression models were also used to estimate the relationship of effective diameter (independent variable) with BMI (dependent variable). A $p$-value lower than 0.05 was taken to indicate statistical significance.

\section{Results \\ Patient demographics}

The study population $(n=50)$ comprised 19 men and 31 women with an age of $37.9 \pm 14.4$ years (mean \pm standard deviation $[\mathrm{SD}]$, ranging from 17 to 73 years.

\section{BMI and dose metrics}

The overall BMI was $24.6 \pm 4.8 \mathrm{~kg} / \mathrm{m}^{2}$ (mean $\left.\pm \mathrm{SD}\right)$, ranging from 17.4 to $38.8 \mathrm{~kg} / \mathrm{m}^{2}$. The mean CTDI $_{\mathrm{vol}}$, DLP, SSDE and effective dose measurements overall, and when stratified for BMI, are listed in Table 1.

\section{Body diameter measurements}

The overall $D_{A P} D_{L A T}, D_{A P+L A T}$ and $D_{E}$ were $24.8 \pm$ $4.5 \mathrm{~cm}, 31.5 \pm 3.9 \mathrm{~cm}, 56.3 \pm 7.9 \mathrm{~cm}$, and $27.8 \pm 4.1 \mathrm{~cm}$ (mean $\pm \mathrm{SD}$ ), respectively. The effective diameter ratio $\left(D_{R A T I O}\right)$ was $1.23 \pm 0.13$ (mean $\pm \mathrm{SD}$ ). The correlations between BMI and diameter are shown in Fig. 2. Overall, the best correlation was found with $D_{E}(0.88)$, where correlations between mean BMI and mean body diameters were highly significant $(p<0.001)$.

Mean BMI and body diameters across BMI subgroups are shown in Table 2 . Excluding $D_{R A T I O}$, the other body

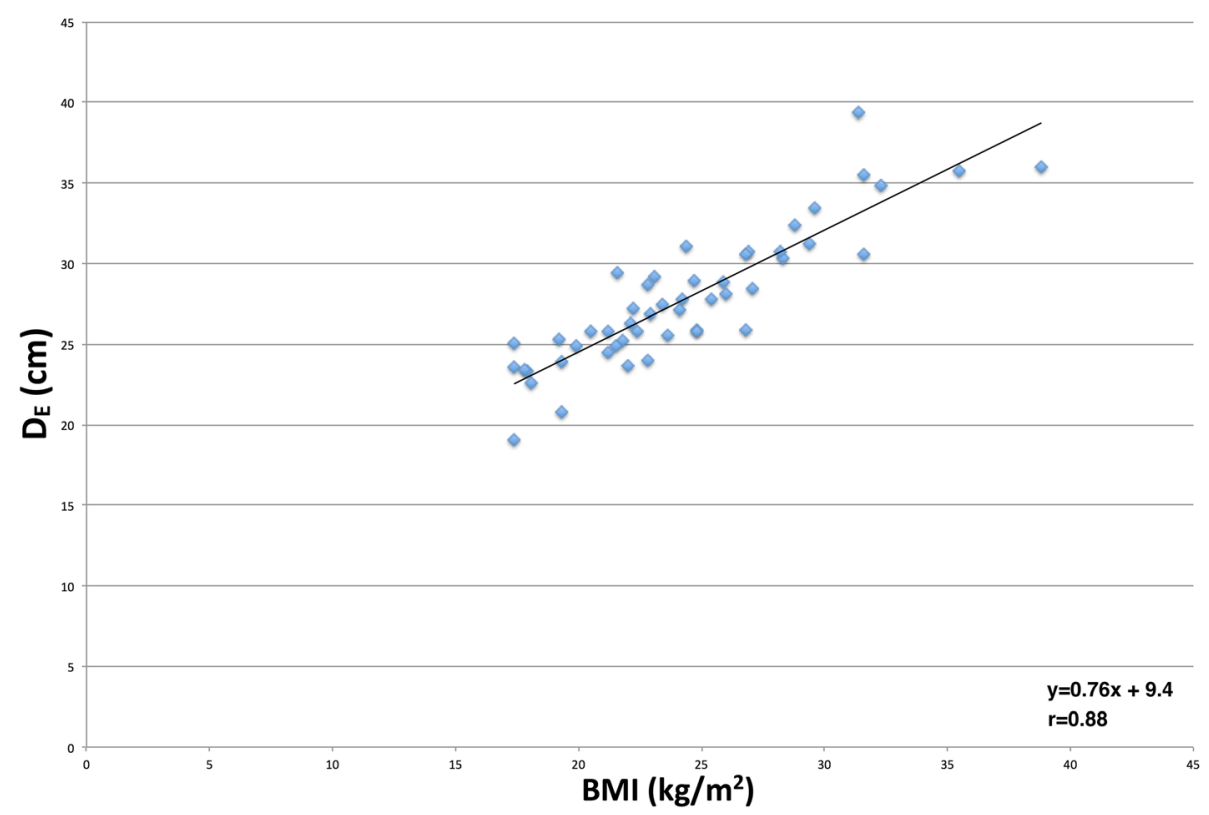

Fig. 4 Scatterplot of body mass index (BMI) as a function of effective diameter. Linear regression trend line equation: $D_{E}=0.76(B M I)+9.4$ 
diameters strongly correlated with each other. The correlation coefficients $(r)$ were 0.78 for $D_{A P}-D_{L A T}, 0.96$ for $D_{A P}-D_{E}$, and 0.92 for $D_{L A T}-D_{E},(p<0.001$ for all). There was a moderate correlation between $D_{\text {LAT }}$ and $\mathrm{D}_{\text {RATIO }}$ with a correlation coefficient of $0.62(p<0.001)$ and weak but statistically significant correlations between $D_{A P}$ and $D_{R A T I O}(r=0.34, p=0.016)$ and $D_{E}$ and $D_{R A T I O}(r=0.49, p<0.001)$. A stronger correlation was found between $D_{A P+L A T}$ and $D_{E}(r=0.99, p<0.001)$ than with either $D_{A P}$ or $D_{L A T}$ alone and $D_{E}[5]$.

Both BMI and effective body diameter correlated strongly with all the dose metrics analysed $(p<0.001)$, with $r$ values ranging from 0.84 to 0.90 . A weaker but significant correlation of $D_{R A T I O}$ with each dose metric was found: $r$ values ranged from 0.45 to $0.48(p \leq 0.05)$. These data are summarised in Fig. 3.

The equation of the linear regression trend-line plotting effective diameter as a function of BMI is as follows: $y=0.76(x)+9.4$ (Fig. 4). Utilising this equation, the patient's BMI $(x)$ can be used to calculate an estimate for the effective diameter $(y)$, i.e. $D_{E}=0.76(\mathrm{BMI})+9.4$. Table 3 demonstrates a list of conversion factors to calculate SSDE from CTDI $_{\text {vol }}$ based on the BMI of the patient.

\section{Discussion}

$\mathrm{CTDI}_{\mathrm{vol}}$ indicates the amount of radiation delivered by the scanner for a specific CT examination, calculated on the basis of a standardised and homogenous phantom study. It is a precisely defined metrics that is displayed on the dose protocol of every CT scanner and is a measure of scanner radiation output, indicating how much radiation is directed toward the patient rather than quantifying how much radiation a patient receives [2]. Increasing use of $\mathrm{CT}$ and concerns regarding radiation dose from medical imaging, however, increase the need for imaging providers to facilitate accurate estimation of radiation dose to patients.

SSDE is a dose parameter that takes into consideration corrections based on the size of the patient from linear dimensions measured on the patient images. SSDE is an estimate of the mean dose to the centre of the scan volume for an object having similar attenuation characteristics as a given patient; it is not a direct measurement of dose to a specific patient [8]. This metrics provides the ability to estimate the average radiation dose to a patient in a clinical setting, albeit with $10-20 \%$ variability of the dose estimate from the actual received dose, even when patient size is taken into account [3]. The AAPM report [3] recommends that the SSDE for each patient be estimated prior to CT scan using patient size parameters to best optimise the scanning parameters to achieve the diagnostic quality CT images with the lowest necessary radiation dose.
Table 3 Conversion factors to convert volume-computedtomography dose index (CTDI vol $_{\text {li }}$ to size-specific dose estimate (SSDE) based on body mass index (BMI)

\begin{tabular}{|c|c|c|}
\hline $\mathrm{BMl}$ & $D_{E}$ & $\mathrm{CTDI}_{\text {vol }} / \mathrm{SSDE}$ conversion factor \\
\hline 15 & 20.8 & 1.73 \\
\hline 16 & 21.6 & 1.68 \\
\hline 17 & 22.3 & 1.63 \\
\hline 18 & 23.1 & 1.59 \\
\hline 19 & 23.8 & 1.54 \\
\hline 20 & 24.6 & 1.50 \\
\hline 21 & 25.4 & 1.46 \\
\hline 22 & 26.1 & 1.42 \\
\hline 23 & 26.9 & 1.38 \\
\hline 24 & 27.6 & 1.34 \\
\hline 25 & 28.4 & 1.31 \\
\hline 26 & 29.2 & 1.27 \\
\hline 27 & 29.9 & 1.23 \\
\hline 28 & 30.7 & 1.20 \\
\hline 29 & 31.4 & 1.17 \\
\hline 30 & 32.2 & 1.14 \\
\hline 31 & 33.0 & 1.10 \\
\hline 32 & 33.7 & 1.07 \\
\hline 33 & 34.5 & 1.04 \\
\hline 34 & 35.2 & 1.02 \\
\hline 35 & 36.0 & 0.99 \\
\hline 36 & 36.8 & 0.96 \\
\hline 37 & 37.5 & 0.93 \\
\hline 38 & 38.3 & 0.91 \\
\hline 39 & 39.0 & 0.88 \\
\hline 40 & 39.8 & 0.86 \\
\hline 41 & 40.6 & 0.84 \\
\hline 42 & 41.3 & 0.81 \\
\hline 43 & 42.1 & 0.79 \\
\hline 44 & 42.8 & 0.77 \\
\hline 45 & 43.6 & 0.75 \\
\hline 46 & 44.4 & 0.73 \\
\hline 47 & 45.1 & 0.71 \\
\hline 48 & 45.9 & 0.69 \\
\hline 49 & 46.6 & 0.67 \\
\hline 50 & 47.4 & 0.65 \\
\hline
\end{tabular}

$B M I$ body mass index, $C T D I_{\text {vol }}$ volume-computed-tomography dose index, $D_{E}$ effective diameter, SSDE size-specific dose estimate

Khawaja et al. [6] demonstrated body weight to be a more simple and convenient measure than effective diameter to estimate SSDE in paediatric patients at the time of CT scanning. They argued that measurement of body diameters in clinical practice is awkward, inconvenient, 
time-consuming and open to interobserver variability, particularly in the absence of a automated methods of measurement. An overview of other attempts to estimate the radiation dose in CT is presented in Table 4 [9-13]. We hypothesised that BMI, being a composite measurement derived from both weight and height, may also represent an appropriate indicator of patient size to use in lieu of effective diameter in predicting SSDE. BMI is an easily, and often routinely, obtained measurement in clinical practice. It is an objective measure with limited bias or interobserver variability during calculation [14].

The present study assessed relevant size metrics and demonstrated a very strong correlation between effective diameter and $\operatorname{SSDE}(r=0.88$ or $0.87 ; p<0.001)$ with $\mathrm{BMI}$, indicating that BMI is an accurate alternative to effective diameter for SSDE estimation in abdominal CT.

Bias from other sources was minimised as all patients were scanned using a predefined and standardised CT abdomen and pelvis protocol on a single CT scanner by one of two radiographers. This paper demonstrates that effective diameter can be accurately estimated using an equation and patient BMI. SSDE can then be computed in a standard manner from $\mathrm{CTDI}_{\mathrm{vol}}$ by using AAPM look-up tables to derive conversion factors.

We found that in patients with a wide range of body habitus measurements (BMI range, 17.4-38.8; effective diameter range, 19-39.4), BMI measurements correlated strongly with diameters. The use of BMI to calculate SSDE has been shown to be a valid alternative to the traditional methods for manual measurement of anterior-posterior and lateral-lateral dimensions using the electronic callipers available on the PACS. A recent study by Babak Alikhani et al. [15] showed that in abdominal CT, the size-dependent conversion factor (f size) closely correlated with patient BMI, indicated by the exponentially decreasing $f$ size values with increasing BMI. The current study echoes these results with the proposition that BMI can act as a surrogate for determining effective body diameter.

The effective diameter ratio $\left(D_{\text {RATIO }}\right)$ is a new metrics proposed by Lamoureux et al. [7] as a supplement to patient-specific size parameter data and is, as yet, not validated. $D_{R A T I O}$ provides information about anatomical composition, particularly the volume of extra-abdominal adipose tissue but underestimates intra-abdominal adiposity. It proved to be a much weaker predictor of radiation dose to the patient in terms of CTDI ${ }_{\mathrm{vol}}$, DLP and effective dose than either effective diameter or BMI $(p<0.001)$ in the present paper. Findings suggest that while this metrics is a good indicator of body fat distribution, it is suboptimal as a predictor of patient SSDE.

Table 4 Examples of dose estimation on abdominal computed tomography (CT)

Monte Carlo dose estimation with patient-specific anatomical models [9]

Automated measurement of effective diameter [10]

Patient size modelled as a water-equivalent diameter $\left(D_{W}\right)[11]$

Dose estimation through directly using thermoluminescent dosemeters (TLDs) [12]

Topogram-based body size indices for $C T$ dose consideration and scan protocol optimisation [13]

Correlating body weight with diameter for radiation dose estimates [6]
Full-body computer model created based on the patient's clinical CT data. Large organs individually segmented and modelled. Other organs were created by transforming an existing adult male or female full-body computer model to match the framework defined by the segmented organs, referencing the organ volume and anthropometry data in ICRP Publication 89. A Monte Carlo program (General Electric Lightspeed VCT-XTe, GE Healthcare, GE Medical Systems, Waukesha, WI, USA) was used to estimate patient-specific organ dose, from which effective dose and risks of cancer incidence were derived. Study suggests the construction of a large library of patient-specific computer models could estimate dose for any patient prior to or after a $C T$ examination

Algorithm for estimating body-size diameter on axial CT slice implemented in Python and C\#. Number of pixels whose Hounsfield unit exceeding a set threshold multiplied by the area of a single pixel to give an estimate of the area of the patient cross-section. Effective diameter computed as diameter of the circle whose area is the same as that of the cross-section. Correlation between the manual and automated measurements of effective diameter was very high

Water-equivalent diameter $\left(D_{w}\right)$, automatically extracted from axial $C T$ images and used to model patient size and subsequently to calculate size-specific dose estimates. The extracted $D_{W}$ values correlate well with effective diameter $\left(R^{2}\right.$ of 0.90 for abdomen and pelvis)

Thermoluminescent dosemeters (TLDs) and a Rando Alderson phantom used. Computersimulated dose estimation based on National Radiation Protection Board Monte Carlo simulations. Directly measured dose $18 \%$ higher than computer-simulated dosimetry, suggesting underestimation by computer-simulation techniques compared with TLD measurements

Linear regression of four topographical indices for estimation of $D_{w}$ (i) average diameter; (ii) girth (cross-section modelled as ellipse); (iii) topogram projection area; (iv) improved topogram projection area (corrected for patient miscentering and water attenuation coefficient)

Anteroposterior and lateral diameters were measured manually and through automated software. Effective diameter subsequently calculated. Overall body weight had a strong correlation with diameter 
Our study has some limitations. Our sample size is relatively low $(n=50)$, with patients chosen because standardised CT imaging on this well-characterised cohort had already been performed and BMI measurements were available for all of them; CT images and data were readily available to test this hypothesis without the need to image further patients. A larger sample size may strengthen the assessment of the relationship between effective diameter and BMI. In addition, with a larger sample size, it could be possible to estimate trend lines stratified for BMI, which may lead to better estimates of diameter and hence of SSDE.

Due to the retrospective design of this study, BMI measurements only were available rather than the constituent height and weights. Statistical evaluation of the interplay of these parameters with BMI, body diameters and radiation dose may have yielded further supportive information. With the advent of automated body diameter and SSDE measurement technology, the applicability of our findings may not be relevant to the small proportion of centres that possess these technologies.

Dose optimisation is a key factor to current radiology practice, particularly for $\mathrm{CT}$ when the correct balance between radiation dose and image quality needs to be struck. To be useful and effective, any applied method of estimating patient radiation dose needs to be userfriendly and reproducible. The present paper indicates that patient BMI can be used to accurately estimate effective diameter, obviating the need to measure anteroposterior and lateral diameters in order to calculate SSDE at the time of CT.

\begin{abstract}
Abbreviations
AAPM: American Association of Physicists in Medicine; BMI: Body mass index; $C T$ : Computed tomography; $C T D_{\text {vol }}$ : Volume $C T$ dose index; $D_{A P}$ : Maximum skin-to-skin anteroposterior diameter; $D_{E}$ : Effective diameter; $D_{I N}$ : Inner $D_{E ;}$ $D_{L A T}$ : Maximum skin-to-skin lateral diameter; DLP: Dose length product; $D_{\text {OUT: }}$ Outer $D_{E} ; D_{\text {RATIO}}$ : Ratio between the internal and the external abdominal diameters; PACS: Picture-archiving and communication system; SD: Standard deviation; SSDE: Size-specific dose estimate
\end{abstract}

\section{Availability of data and materials}

The datasets used and/or analysed during the current study are available from the corresponding author on reasonable request.

\section{Funding}

Professor Denis O'Sullivan Research Fellowship, University College Cork, Cork, Ireland.

\section{Authors' contributions}

SON is involved with study design, data acquisition and analysis, manuscript drafting and revising. RGK is involved with data analysis, manuscript drafting and revising. BWC is involved with data analysis, manuscript drafting and revising. NM is involved with study design, data acquisition, manuscript drafting and revising. MM is involved with study design, manuscript drafting and revising. OJOC is involved with study design, data analysis, manuscript drafting and revising. All authors have given final approval to manuscript publication and agree to be accountable for all aspects of the work in ensuring that questions related to the accuracy or integrity of any part of the work are appropriately investigated and resolved.
Ethics approval and consent to participate

Approval for this study was granted by the Clinical Research Ethics Committee of the Cork Teaching Hospitals, Lancaster Hall, 6 Little Hanover Street, Cork, Ireland. Informed consent was obtained from each patient.

\section{Consent for publication}

The consent for publication is included in the informed consent.

\section{Competing interests}

The authors declare that they have no competing interests.

\section{Publisher's Note}

Springer Nature remains neutral with regard to jurisdictional claims in published maps and institutional affiliations.

\section{Author details}

${ }^{1}$ Department of Radiology, Cork University Hospital, Wilton, Cork, Ireland.

${ }^{2}$ Department of Radiology, University College Cork, Cork, Ireland. ${ }^{3}$ APC

Microbiome Ireland, University College Cork, Cork, Ireland.

Received: 18 June 2018 Accepted: 9 October 2018

Published online: 28 November 2018

References

1. Brenner DJ, McCollough C, Orton CG (2006) Is it time to retire the computed tomography dose index (CTDI) for CT quality assurance and dose optimisation? Med Phys 33:1189-1190

2. McCollough CH, Leng S, Yu L, Cody DD, Boone JM, McNitt-Gray MF (2011) CT dose index and patient dose: they are not the same thing. Radiology 259:311-316

3. American Association of Physicists in Medicine (2011) Size-specific dose estimates (SSDE) in pediatric and adult body CT examinations (Report 204). https://www.aapm.org/pubs/reports/RPT_204.pdf. Accessed Sept 222018

4. American Association of Physicists in Medicine (2014) Use of water equivalent diameter for calculating patient size and size-specific dose estimates (SSDE) in CT (Report 220). https://www.aapm.org/pubs/reports/RPT_220.pdf. Accessed Sept 222018

5. Craig O, O' Neill S, O'Neill F et al (2012) Diagnostic accuracy of computed tomography using lower doses of radiation for patients with Crohn's disease. Clin Gastroenterol Hepatol 10:886-892

6. Khawaja RD, Singh S, Vettiyil B et al (2015) Simplifying size-specific radiation dose estimates in pediatric CT. AJR Am J Roentgenol 204:167-176

7. Lamoureux R, Sinclair L, Mench A et al (2015) SU-E-I-28: introduction and investigation of effective diameter ratios as a new patient size metric for use in CT. Med Phys 42:3247-3248

8. Christner JA, Braun NN, Jacobsen MC, Carter RE, Kofler JM, McCollough CH (2012) Size-specific dose estimates for adult patients at CT of the torso. Radiology 265:841-847

9. Li X, Samei E, Segars WP et al (2011) Patient-specific radiation dose and cancer risk estimation in CT: part II. Application to patients. Med Phys 38:408-419

10. Cheng PM (2013) Automated estimation of abdominal effective diameter for body size normalization of CT dose. J Digit Imaging 26:406-411

11. Ikuta I, Warden Gl, Andriole KP, Khorasani R, Sodickson A (2014) Estimating patient dose from $x$-ray tube output metrics: automated measurement of patient size from $C T$ images enables large-scale size-specific dose estimates. Radiology 270:472-480

12. Groves AM, Owen KE, Courtney HM et al (2004) 16-detector multislice CT: dosimetry estimation by TLD measurement compared with Monte Carlo simulation. Br J Radiol 77:662-665

13. Li B, Behrman RH, Norbash AM (2012) Comparison of topogram-based body size indices for $\mathrm{CT}$ dose consideration and scan protocol optimization. Med Phys 39:3456-3465

14. Sebo P, Beer-Borst S, Haller DM, Bovier PA (2008) Reliability of doctors' anthropometric measurements to detect obesity. Prev Med 47:389-393

15. Alikhani B, Getzin T, Kaireit TF et al (2018) Correlation of size-dependent conversion factor and body-mass-index using size-specific dose estimates formalism in CT examinations. Eur J Radiol 100:130-134 